\title{
Can Computed Tomographic Angiography Be Used to Predict Who Will Not Benefit from Endovascular Treat- ment in Patients with Acute Ischemic Stroke? The CTA- ABC Score
}

\author{
Hyo-Sung Kwak, Jung-Soo Park ${ }^{2}$ \\ Department of Radiology and Research Institute of Clinical Medicine of Jeonbuk National University-Biomedical Research Institute of Jeonbuk \\ National University Hospital,' Jeonju, Korea \\ Department of Neurosurgery and Research Institute of Clinical Medicine of Jeonbuk National University-Biomedical Research Institute of \\ Jeonbuk National University Hospital, ${ }^{2}$ Jeonju, Korea
}

Objective : The objective of this study was to develop a score to predict patients with acute ischemic stroke (AIS) who will not benefit from endovascular treatment (EVT) using computed tomographic angiography (CTA) parameters.

Methods : The CTA-ABC score was developed from 3 scales previously described in the literature: the Alberta Stroke Program Early CT Score ( $0-5$ points, 3; 6-10 points, 0$)$, the clot burden score ( $0-3$ points, $1 ; 4-10$ points, 0$)$, and the leptomeningeal Collateral score (0-1 points, 2; 2-3 points, 0 ). We evaluated the predictive value of CTA parameters associated with symptomatic intracranial hemorrhage (sICH) or malignant middle cerebral artery infarction (MMCAl) after EVT and developed the score using logistic regression coefficients. The score was then validated. Performance of the score was tested with an area under the receiver operating characteristic curve (AUC-ROC).

Results : The derivation cohort consisted of 115 and the validation cohort consisted of 40 AIS patients. The AUC-ROC was 0.97 (95\% confidence interval [Cl], $0.94-0.99 ; p<0.001)$ in the derivation cohort. The proportions of patients with sICH and/or MMCAl in the derivation cohort were $96 \%, 73 \%, 6 \%$, and $0 \%$ for scores of $6,5,1$, and 0 points, respectively. In the validation group, the proportions were similar (90\%, 100\%, 0\%, and 0\%, respectively) with an AUC-ROC of $0.96(95 \% \mathrm{Cl}, 0.90-1.00 ; p<0.001)$.

Conclusion : Our CTA-ABC score reliably assessed risk for sICH and/or MMCAI in patients with AIS who underwent EVT. It can support clinical decision-making, especially when the need for EVT is uncertain.

Key Words : Stroke. Cerebral angiography · Thrombectomy.

- Received : October 15, $2019 \cdot$ Revised : November 26, $2019 \bullet$ Accepted : December 2, 2019

- Address for reprints : Jung-Soo Park

Department of Neurosurgery and Research Institute of Clinical Medicine of Jeonbuk National University-Biomedical Research Institute of Jeonbuk National University Hospital, 567 Baekje-daero, Deokjin-gu, Jeonju 561-756, Korea

Tel : +82-63-250-1755, Fax : +82-63-277-3273, E-mail : nspark@jbnu.ac.kr, ORCID : https://orcid.org/0000-0002-4949-203X

This is an Open Access article distributed under the terms of the Creative Commons Attribution Non-Commercial License (http://creativecommons.org/licenses/by-nc/4.0) which permits unrestricted non-commercial use, distribution, and reproduction in any medium, provided the original work is properly cited. 


\section{INTRODUCTION}

The efficacy and safety of endovascular treatment (EVT) as compared to medical treatment in patients with acute ischemic stroke (AIS) caused by anterior circulation large vessel occlusion (LVO) has been well documented ${ }^{1,2,6,7)}$. According to previous studies, EVT in patients with acute LVO in the anterior circulation results in high rates of successful recanalization (thrombolysis in cerebral infarction score 2 b or $3 ; 72.4-$ $86 \%)$. Nevertheless, even with such high recanalization rates, only 33 to $54 \%$ of patients show good clinical outcomes (modified Rankin scale [mRS] score 0-2), and 12 to $30 \%$ of patients may have resultant severe disability or death ( $\mathrm{mRS} 5$, $6)^{1,2,6,7)}$

Symptomatic intracranial hemorrhage (sICH) and malignant middle cerebral artery infarction (MMCAI) may negate the efficacy of EVT in patients with anterior circulation LVO. Therefore, identifying the predictors for sICH or MMCAI after EVT may help predict prognosis and guide early patient management. Computed tomographic angiography (CTA) is the modality used to confirm vessel occlusion prior to EVT. As compared to magnetic resonance imaging (MRI), CTA can identify the occluded vessel and can exclude hemorrhagic stroke in a timely manner.

The aim of our study was to develop a prediction score to determine who would not benefit from EVT using easily assessable CTA parameters in patients with AIS caused by anterior circulation LVO.

\section{MATERIALS AND METHODS}

This retrospective study was approved by the Institutional Review Board of Jeonbuk National University Hospital.

\section{Derivation and validation Cohort}

We used a prospectively collected clinical and radiological database to enroll patients at our institution who presented with AIS treated with EVT between January 2015 and September 2018. Our study targeted patients who received CTA at the time of admission with a documented anterior circulation LVO caused by an internal carotid artery (ICA) occlusion. After establishing the score, we validated it in eligible patients with AIS treated with EVT at our institution between October
2018 to April 2019. All eligible patients received $0.9 \mathrm{mg} / \mathrm{kg}$ of intravenous recombinant tissue plasminogen activator within 4.5 hours of symptom onset.

\section{Image acquisition}

After excluding hemorrhagic stroke through noncontrast computed tomography (NCCT), CTA was performed with a multislice CT scanner (SOMATOM Sensation 16; Siemens, Erlangen, Germany) with images acquired from the vertex to the aortic arch. Nonionic contrast media $(80-120 \mathrm{~mL})$ was administered into the antecubital vein at $3-5 \mathrm{~mL} / \mathrm{s}$. The images were autotriggered by the appearance of contrast media in the superior sagittal sinus, and the CTA source image was reconstructed in $1 \mathrm{~mm}$ thickness slices in the axial planes at $0.7 \mathrm{~mm}$ thickness intervals. NCCT was performed immediately after EVT and on follow-up at 24 hours.

\section{Clinical and image analysis}

The initial neurological status of patients was evaluated using the National Institutes of Health Stroke Scale (NIHSS) score at admission. The mRS was checked on admission and at 3 months after presentation. A favorable functional outcome was defined as a 3 -month mRS score of $\leq 2$. Image review was independently performed on a wide-screen highresolution monitor with adequate window and level settings using 3 scores previously described in the literature. The CTA Alberta Stroke Program Early CT Score (CTA ASPECTS) is calculated by subtracting the number of diminished contrast enhancement regions from a total score of 10 on the CTA source image. The clot burden score (CBS) is a quantified assessment of the extent of the clot in the anterior circulation. Starting at 10, points are deducted based on the loss of contrast opacification on CTA. Two points are subtracted for contrast opacification within the supraclinoid ICA, the proximal M1 branch of the middle cerebral artery (MCA), and the distal M1 of the MCA. One point is subtracted for the infraclinoid ICA, both M2 branches of the MCA, and the A1 segment of anterior cerebral artery ${ }^{11)}$. The leptomeningeal collateral status score is assessed on a scale of $0-3$. A score of 0 indicates absent collateral supply; a score of 1 indicates collateral supply filling $\leq 50 \%$; a score of 2 indicates $>50 \%$ but $<100 \%$ collateral supply filling, and a score of 3 indicates $\geq 100 \%$ collateral supply filling as compared to the non-occluded side ${ }^{13)}$. 


\section{Definition of MMCAI and sICH}

An MMCAI was defined as having the following : 1) NIHSS score $>18$; 2) large space-occupying MCA infarction on follow-up CT or MRI with at least two-thirds of the MCA territory demonstrating compression of the ventricle or midline shift; and 3) no other clear cause for neurological deterioration $^{14)}$. The sICH was defined as an intracranial hemorrhage associated with an NIHSS $\geq 4$ between the postprocedural as- sessment and the 24-hour follow up ${ }^{15)}$.

\section{Statistics analysis}

We performed a univariate comparison of groups in both cohorts using an independent t-test for continuous variables and a Pearson chi-square test for categorical variables. We used multivariate regression to estimate the predictive value of the CTA parameters associated with MMCAI or sICH after

Table 1. Demographics and baseline characteristics of the patients

\begin{tabular}{|c|c|c|c|}
\hline & Derivation cohort $(n=115)$ & Validation cohort $(n=40)$ & $p$-value \\
\hline Mean age (years) & $72.7 \pm 11.3$ & $74.1 \pm 10.4$ & 0.508 \\
\hline Men & $66(57)$ & $28(70)$ & 0.160 \\
\hline Baseline NIHSS score & $12(10-15)$ & $15(10-17)$ & 0.170 \\
\hline Baseline CTA ASPECTS & $8(3-9)$ & $7(4-8)$ & 0.817 \\
\hline Baseline CBS & $5(2-7)$ & $4(1-7)$ & 0.437 \\
\hline Baseline collateral circulation score & $2(1-2)$ & $1(1-2)$ & 0.547 \\
\hline Onset to door time (minutes) & $100(60-160)$ & $124(68-240)$ & 0.145 \\
\hline \multicolumn{4}{|l|}{ Risk factors } \\
\hline Diabetes & $23(20)$ & $10(25)$ & 0.506 \\
\hline Hypertension & $61(53)$ & $21(53)$ & 0.953 \\
\hline Hyperlipidemia & $9(8)$ & $1(3)$ & 0.238 \\
\hline Smoking & $23(20)$ & $12(30)$ & 0.193 \\
\hline Previous stroke & $26(23)$ & 7 (18) & 0.497 \\
\hline Atrial fibrillation or cardiac disease & $42(37)$ & $15(38)$ & 0.912 \\
\hline Intravenous tPA use & $98(85)$ & $33(83)$ & 0.682 \\
\hline
\end{tabular}

Values are presented as mean \pm standard deviation, number (\%), or median (interquartile range). NIHSS : National Institutes of Health Stroke Scale, CTA : computed tomographic angiography, ASPECTS : Alberta Stroke Program Early CT score, CBS : clot burden score, tPA : tissue plasminogen activator

Table 2. Baseline characteristics, including CTA parameters and clinical outcomes, of derivation patients according to occurrence of sICH and/or MMCAI

\begin{tabular}{|c|c|c|c|}
\hline Variable & sICH(+) and/or MMCAI(+) $(n=36)$ & sICH(-) and MMCAI (-) (n=79) & $p$-value \\
\hline Mean age (years) & $71.1 \pm 12.7$ & $73.5 \pm 10.6$ & 0.305 \\
\hline Baseline NIHSS score & $14(11-17)$ & $12(9-14)$ & 0.001 \\
\hline Baseline CTA ASPECTS & $3(2-4)$ & $8(7-9)$ & $<0.001$ \\
\hline Baseline CBS & $1(1-4)$ & $6(3-7)$ & $<0.001$ \\
\hline Baseline collateral circulation score & $1(0-1)$ & $2(2-2)$ & $<0.001$ \\
\hline Onset to recanalization time (minutes) & $272(208-350)$ & $230(177-348)$ & 0.382 \\
\hline $\mathrm{TICl} \geq 2 \mathrm{~b}$ & $26(72)$ & $58(73)$ & 0.893 \\
\hline Discharge NIHSS score & $14(9-20)$ & $6(4-10)$ & $<0.001$ \\
\hline Favorable functional outcome (mRS 0-2) & $4(11)$ & $26(33)$ & 0.014 \\
\hline
\end{tabular}

Values are presented as mean \pm standard deviation, number (\%), or median (interquartile range). CTA : computed tomographic angiography, sICH : symptomatic intracranial hemorrhage, MMCAl : malignant middle cerebral artery infarction, NIHSS : National Institutes of Health Stroke Scale, ASPECTS : Alberta Stroke Program Early CT score, CBS : clot burden score, $\mathrm{TICl}$ : thrombolysis in cerebral infarction, mRS : modified Rankin scale 
EVT in the derivation cohort. The scoring system was developed according to the magnitude of regression coefficients.

The multivariate model used in our study was as follows : 1) selection of independent variables. We included those CTA variables determined to have been associated with sICH or MMCAI from previous studies ${ }^{3,5,15}$. We analyzed the area under the receiver operating characteristic curve (AUC-ROC) to determine the best cut-off point of the CTA parameters to identify patients with sICH and/or MMCAI after EVT in the derivation cohort. 2) CTA-ABC score. Using multiple logistic regression analysis, we confirmed that the CTA parameter cut-off values were independent predictors for sICH and/or MMCAI. We then developed the CTA-ABC score (which combined the CTA ASPECTS, the CBS, and the leptomeningeal Collateral score) according to the magnitude of the logistic regression coefficients. And 3) the score was validated in the validation cohort using an AUC-ROC.
We considered an AUC-ROC $>0.75$ as an acceptable value with statistical significance defined as a $p<0.05$. Statistical analyses were performed using SPSS ver. 23.0 (IBM SPSS, Chicago, IL, USA).

\section{RESULTS}

During the study time period, 488 AIS patients were treated with EVT. Of this number, 373 patients were ineligible for the study; this included 252 patients that did not have a related ICA occlusion, 52 patients without an available baseline CTA of adequate quality to evaluate the CTA parameters, and 69 patients without an initial CTA. The final CTA-ABC derivation cohort was therefore composed of 115 patients. Our validation cohort consisted of 40 eligible patients with AIS treated with EVT.

Table 3. AUC-ROC analysis of CTA and NCCT ASPECTS cutoff value for symptomatic hemorrhage(-) and malignant MCA infarction(-)

\begin{tabular}{lccccc}
\hline & AUC $(\mathbf{9 5} \% \mathrm{Cl})$ & $\boldsymbol{p}$-value & Best cutoff value & Sensitivity (\%) & Specificity (\%) \\
\hline CTA ASPECTS & $0.98(0.96-1.00)$ & $<0.001$ & 6 & 94.9 & 88.9 \\
LMC score & $0.92(0.87-0.97)$ & $<0.001$ & 2 & 75.9 & 97.2 \\
CBS & $0.80(0.71-0.89)$ & $<0.001$ & 4 & 73.4 & 72.2 \\
\hline
\end{tabular}

AUC-ROC : area under the receiver operating characteristic curve, CTA : computed tomographic angiography, NCCT : noncontrast computed tomography, ASPECTS : Alberta Stroke Program Early CT score, MCA : middle cerebral artery, Cl : confidence interval, LMC : leptomeningeal collateral, CBS : clot burden score

Table 4. Variables associated with symptomatic hemorrhage (+) and/or malignant MCA infarction(+) after EVT

\begin{tabular}{|c|c|c|c|c|}
\hline & Univariate analysis & $p$-value & Multivariate analysis & $p$-value \\
\hline Age $>80$ years & $1.61(0.66-3.91)$ & 0.297 & & \\
\hline Baseline NIHSS score $\geq 15$ & $2.42(1.04-5.64)$ & 0.041 & $2.36(0.46-11.99)$ & 0.302 \\
\hline Onset to recanalization $\geq 6$ hours & $2.30(0.44-12.01)$ & 0.322 & & \\
\hline CTA ASPECTS $\leq 5$ & $150.00(35.31-637.15)$ & $<0.001$ & 36.39 (6.39-207.29) & $<0.001$ \\
\hline LMC score $\leq 1$ & 110.53 (14.12-861.69) & $<0.001$ & $11.13(1.02-121.86)$ & 0.048 \\
\hline $\mathrm{CBS} \leq 3$ & $7.18(2.87-17.38)$ & $<0.001$ & $4.84(1.01-23.17)$ & 0.049 \\
\hline Diabetes & $1.55(0.60-4.00)$ & 0.368 & & \\
\hline Hypertension & $1.04(0.46-2.24)$ & 0.913 & & \\
\hline Hyperlipidemia & $0.61(0.12-3.07)$ & 0.544 & & \\
\hline Smoking & $0.95(0.35-2.56)$ & 0.920 & & \\
\hline Atrial fibrillation & $0.38(0.15-0.93)$ & 0.335 & & \\
\hline Previous stroke history & $1.88(0.76-4.64)$ & 0.273 & & \\
\hline
\end{tabular}

Values are presented as odds ratio (95\% confidence interval). MCA : middle cerebral artery, EVT : endovascular treatment, NIHSS : National Institutes of Health Stroke Scale, CTA : computed tomographic angiography, ASPECTS : Alberta Stroke Program Early CT score, LMC : leptomeningeal collateral, CBS : clot burden score 
The demographics and baseline characteristics of patients in the derivation $(n=115)$ and validation $(n=40)$ cohorts are shown in Table 1. There were no significant differences between the two groups. The prevalence of sICH and/or MMCAI infarction in the derivation cohort was 36\%. The baseline characteristics, including CTA parameters and clinical outcomes, of the patients classified according to the occurrence of sICH and/or MMCAI are presented in Table 2. The patients presenting with sICH and/or MMCAI showed statistically significantly lower ASPECTS, CBS and collateral circulation scores on baseline CTA. The AUC-ROC analysis was performed to find the best cutoff value of CTA parameters to predict occurrence of sICH and MMCAI. The CTA parameters with good performance for prediction of patients with

Table 5. CTA-ABC score (0-6) accessing risk for sICH and/or MMCAI in acute ischemic stroke patients undergoing endovascular treatment

\begin{tabular}{lccc}
\hline CTA parameter & Points & Regression coefficient & $p$-value \\
\hline CBS & & & \\
$0-3$ points & 1 & Reference & \\
$\geq 4$ points & 0 & & \\
LMC score & & 2.48 & 0.049 \\
$0-1$ point & 2 & Reference & \\
$\geq 2$ points & 0 & & \\
CTA ASPECTS & & 3.59 & $<0.001$ \\
$0-5$ points & 3 & Reference & \\
$\geq 6$ points & 0 & & \\
\hline
\end{tabular}

CTA : computed tomographic angiography, sICH : symptomatic intracranial hemorrhage, MMCAI : malignant middle cerebral artery infarction, CBS : clot burden score, LMC : leptomeningeal collateral, ASPECTS : Alberta Stroke Program Early CT score

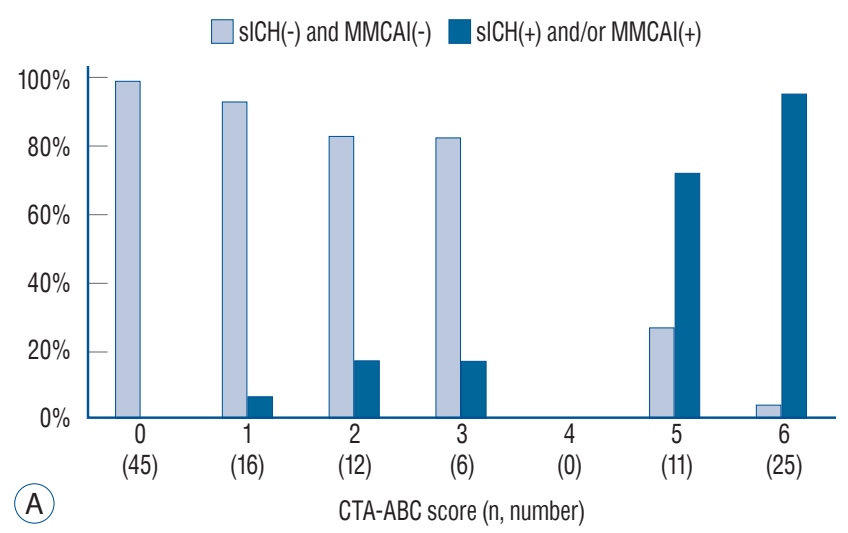

sICH and MMCAI were as follows : 1) CTA ASPECTS of 0.98 (95\% confidence interval [CI], 0.96-100; $p<0.001$ ) and 2 ) collateral score of 0.92 (95\% CI, $0.87-0.97 ; p<0.001)$ and a CBS of 0.80 (95\% CI, 0.71-89; $p<0.001$ ). The best cut-off values for the AUC-ROC analysis were 6 for the CTA ASPECTS score, 2 for the collateral score, and 4 for the CBS (Table 3). Based on these cutoff values, the CTA parameters identified as independent predictors through multivariate analysis for occurrence of sICH and/or MMCAI after EVT were a CTA ASPECTS $\leq 5$, a collateral circulation score $\leq 1$ and a CBS $\leq 3$ (Table 4). Based upon these statistical results, we developed a CTA-ABC score (CTA ASPECTS, CBS and leptomeningeal Collateral score) score with a scale ranging from 0 to 6 according to the magnitude of logistic regression coefficients. Table 5 describes the score in more detail.

The absolute risk for sICH and/or MMCAI after EVT in patients based on the CTA-ABC score is shown in Fig. 1. The proportions of patients with $\mathrm{sICH}$ and/or MMCAI in the derivation cohort were $96 \%, 73 \%, 17 \%, 17 \%, 6 \%$, and $0 \%$ for 6,5 , $3,2,1$, and 0 points, respectively. The performance of the score as estimated with the AUC-ROC for the derivation cohort was 0.97 (95\% CI, 0.94-0.99; $p<0.001$ ). In the validation cohort, the proportions of patients with sICH and/or MMCAI were $90 \%, 100 \%, 67 \%, 25 \%, 0 \%, 0 \%$, and $0 \%$, for $6,5,4,3,2,1$, and 0 points, respectively. The performance of the score for the validation cohort was 0.96 (95\% CI, 0.90-1.00; $p<0.001)$ based on the AUC-ROC analysis. The best cutoff value of the CTA-ABC score to predict sICH and/or MMCAI after EVT in the validation cohort was 4 points, with a sensitivity of $88.9 \%$ and a specificity of $94.9 \%$.

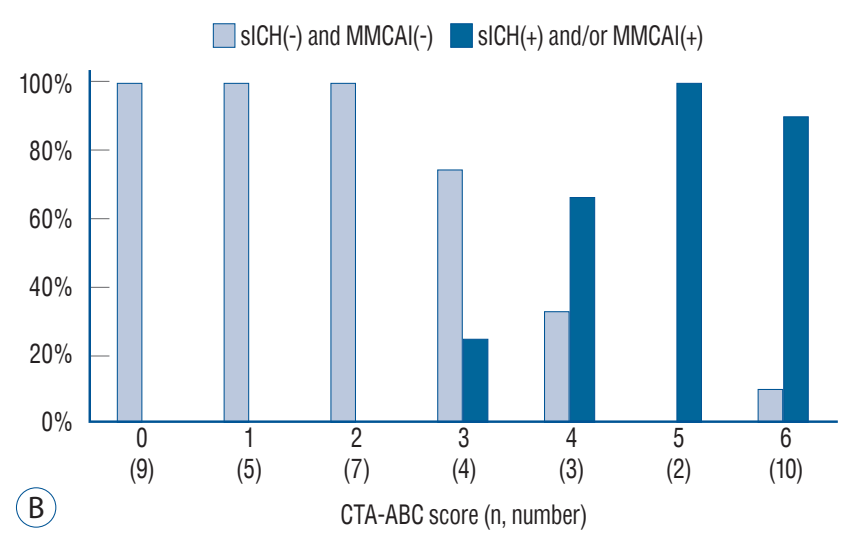

Fig. 1. Proportion of $s I C H$ and/or MMCAI per increase in CTA-ABC score. A : Derivation cohort $(n=115)$. B : Validation cohort $(n=40)$. sICH : symptomatic intracranial hemorrhage, MMCAl : malignant middle cerebral artery infarction, CTA : computed tomographic angiography. 


\section{DISCUSSION}

In recent years, EVT has been considered a safe and effective treatment modality for LVO in the anterior circulation in patients with AIS $^{1,2,6,7)}$. Nevertheless, in some cases, the neurologic status of a patient is worse after treatment. The majority of patients with poor prognoses are those who eventually progress to have an MMCAI or who develop a sICH after the procedure. Therefore, identifying the predictors for MMCAI or sICH after EVT may help identify optimal candidates for EVT. Our study developed a score to help predict such patients who would not benefit from EVT in cases of acute anterior circulation AIS.

Previous studies have demonstrated that the CTA ASPECTS determined using CTA source images is reliably correlated with the ischemic volume and final infarction size identified on magnetic resonance diffusion-weighted imaging ${ }^{4,8)}$. Davoli et al. ${ }^{3)}$ reported that the CTA ASPECTS is an independent predictor of MMCAI evolution in patients with AIS undergoing mechanical thrombectomy; a CTA ASPECTS $\leq 5$ predicted MMCAI evolution with a remarkably high sensitivity (97.14\%). Similarly, in our study, a CTA ASPECTS of 6 was the best cut-off value to predict patients who did not have a subsequent MMACI and sICH with a high sensitivity (94.9\%). A CTA ASPECTS $\leq 5$ was independent predictor of MMCAI and/or sICH evolution after EVT (odds ratio [OR], 36.39; 95\% CI, 6.39-207.29; $p<0.001$ ).

Previous studies have demonstrated that a noninvasive leptomeningeal collateral circulation (CC) scoring system using CTA is well correlated with clinical outcomes in patients with AIS $^{9,10,12)}$. Flores et al. ${ }^{5)}$ reported that the CC score assessed by multiphase CTA independently predicts MMCAI progression. We used a scale of $0-3$ with a scoring system proposed by Tan et al. $^{13)}$ to calculate the CC score. In our study, a CC score of 3 was the best cut-off value to predict patients who did not have a subsequent MMACI and sICH (sensitivity, 75.9\%; specificity, 97.2\%). A CC score $\leq 1$ was an independent predictor of MMCAI and/or sICH evolution after EVT (OR, 11.13; 95\% CI, 1.02-121.86; $p=0.048$ ).

Puetz et al. ${ }^{11)}$ developed the CBS scoring system based on the presence of contrast opacification on CTA. The CBS was not only correlated with the final infarction size and hemorrhagic transformation rate, but was also an independent predictor of clinical outcomes and mortality. Recently, Yogendra- kumar et al. ${ }^{15)}$ reported that post EVT intracranial hemorrhage was associated with a CBS $\leq 3$ (sensitivity, $41 \%$; specificity, 82\%; OR, 3.12; 95\% CI, 1.36-7.15), which robustly predicted hemorrhage. Our results were consistent with these prior findings. In our study, a $\mathrm{CBS} \leq 3$ was an independent predictor of MMCAI and/or sICH evolution (OR, 4.84; 95\% CI, 1.0123.17; $p=0.049$ ).

Based on these statistical results, we developed the CTAABC score (Table 5). The score reliably predicted risk in our sample for sICH and/or MMCAI in patients with anterior circulation LVO treated with EVT (Fig. 1). Moreover, the performance of the score as estimated with the AUC-ROC was 0.97 (0.94-0.99) in the derivation cohort and $0.96(0.90-1.00)$ in the validation cohort.

Our study has a number of limitations. Although we controlled for CTA parameters that were known to be predictors of stroke outcomes, we cannot rule out the possibility that other excluded baseline variables may have had an impact on the development of subsequent MMCAI or sICH. In addition, we were limited by the small size of the derivation cohort used to develop our score. The validation cohort was also small and collected from our institution and therefore may have restricted the generalizability of the performance of the score. Despite these limitations, we believe that this scoring system may be useful in aiding the decision-making process for selection of future EVT candidates, especially considering its performance based on the AUC-ROC of 4 points or more. At this time, although the CTA-ABC score cannot be used to completely exclude candidates for EVT, it may aid in predicting the prognosis after EVT or guide the clinician in the choice of conservative management versus aggressive EVT, especially in cases of uncertainty.

\section{CONCLUSION}

CTA is useful for confirmation of vessel occlusion for EVT, and the CTA-ABC score using CTA parameters is a simple way to predict patients who may not benefit from EVT. Although further external validation of the CTA-ABC score is warranted, it may provide useful information to neurointerventionists about the potential utility of EVT in individual cases. Future research is needed on larger samples to help further evaluate the predictive value of the CTA-ABC score. 


\section{CONFLICTS OF INTEREST}

No potential conflict of interest relevant to this article was reported.

\section{INFORMED CONSENT}

Informed consent was obtained from all individual participants included in this study.

\section{AUTHOR CONTRIBUTIONS}

\author{
Conceptualization : HSK \\ Data curation : HSK, JSP \\ Formal analysis : HSK, JSP \\ Funding acquisition : JSP \\ Methodology : JSP \\ Project administration : HSK, JSP \\ Visualization : JSP \\ Writing - original draft : JSP \\ Writing - review \& editing : HSK, JSP
}

\section{ORCID}

Hyo-Sung Kwak https://orcid.org/0000-0002-7228-8117

Jung-Soo Park https://orcid.org/0000-0002-4949-203X

\section{- Acknowledgements}

This paper was supported by Fund of Biomedical Research Institute, Jeonbuk National University Hospital.

\section{References}

1. Berkhemer $O A$, Fransen PS, Beumer $D$, van den Berg LA, Lingsma HF, Yoo AJ, et al. : A randomized trial of intraarterial treatment for acute ischemic stroke. N Engl J Med 372 : 11-20, 2015

2. Campbell BC, Mitchell PJ, Kleinig TJ, Dewey HM, Churilov L, Yassi N, et al. : Endovascular therapy for ischemic stroke with perfusion-imaging selection. N Engl J Med 372 : 1009-1018, 2015
3. Davoli A, Motta C, Koch G, Diomedi M, Napolitano S, Giordano A, et al. : Pretreatment predictors of malignant evolution in patients with ischemic stroke undergoing mechanical thrombectomy. J Neurointerv Surg $10: 340-344,2018$

4. Ezzeddine MA, Lev MH, McDonald CT, Rordorf G, Oliveira-Filho J, Aksoy FG, et al. : CT angiography with whole brain perfused blood volume imaging: added clinical value in the assessment of acute stroke. Stroke 33 : 959-966, 2002

5. Flores $A$, Rubiera M, Ribó M, Pagola J, Rodriguez-Luna D, Muchada M, et al. : Poor collateral circulation assessed by multiphase computed tomographic angiography predicts malignant middle cerebral artery evolution after reperfusion therapies. Stroke 46 : 3149-3153, 2015

6. Goyal M, Demchuk AM, Menon BK, Eesa M, Rempel JL, Thornton J, et al. : Randomized assessment of rapid endovascular treatment of ischemic stroke. N Engl J Med 372 : 1019-1030, 2015

7. Jovin TG, Chamorro A, Cobo E, de Miquel MA, Molina CA, Rovira A, et al. : Thrombectomy within 8 hours after symptom onset in ischemic stroke. N Engl J Med 372 : 2296-2306, 2015

8. Lev MH, Segal AZ, Farkas J, Hossain ST, Putman C, Hunter GJ, et al. : Utility of perfusion-weighted CT imaging in acute middle cerebral artery stroke treated with intra-arterial thrombolysis: prediction of final infarct volume and clinical outcome. Stroke 32 : 2021-2028, 2001

9. Maas MB, Lev MH, Ay H, Singhal AB, Greer DM, Smith WS, et al. : Collateral vessels on $\mathrm{CT}$ angiography predict outcome in acute ischemic stroke. Stroke 40 : 3001-3005, 2009

10. Menon BK, Smith EE, Modi J, Patel SK, Bhatia R, Watson TW, et al. : Regional leptomeningeal score on $\mathrm{CT}$ angiography predicts clinical and imaging outcomes in patients with acute anterior circulation occlusions.

AJNR Am J Neuroradiol 32 : 1640-1645, 2011

11. Puetz V, Dzialowski I, Hill MD, Subramaniam S, Sylaja PN, Krol A, et al. : Intracranial thrombus extent predicts clinical outcome, final infarct size and hemorrhagic transformation in ischemic stroke: the clot burden score. Int J Stroke 3 : 230-236, 2008

12. Shuaib A, Butcher K, Mohammad AA, Saqqur M, Liebeskind DS : Collateral blood vessels in acute ischaemic stroke: a potential therapeutic target. Lancet Neurol 10 : 909-921, 2011

13. Tan IY, Demchuk AM, Hopyan J, Zhang L, Gladstone D, Wong K, et al. : $\mathrm{CT}$ angiography clot burden score and collateral score: correlation with clinical and radiologic outcomes in acute middle cerebral artery infarct. AJNR Am J Neuroradiol 30 : 525-531, 2009

14. Thomalla G, Hartmann F, Juettler E, Singer OC, Lehnhardt FG, Köhrmann $M$, et al. : Prediction of malignant middle cerebral artery infarction by magnetic resonance imaging within 6 hours of symptom onset: a prospective multicenter observational study. Ann Neurol 68 : 435-445, 2010

15. Yogendrakumar V, Al-Ajlan F, Najm M, Puig J, Calleja A, Sohn SI, et al. : Clot burden score and early ischemia predict intracranial hemorrhage following endovascular therapy. AJNR Am J Neuroradiol 40 : 655660,2019 